\title{
NUEVOS RECURSOS EDUCATIVOS EN EL MARCO EUROPEO: CREADICTOS (FESTIVAL DE CREATIVIDAD UNIVERSITARIO)
}

Laura Melendo Rodríguez-Carmona1. Universidad Camilo José Cela. España. lmelendo@ucjc.edu

Eva García-Montero. Universidad Camilo José Cela. España egarmo@ucjc.edu

\section{RESUMEN}

El aprendizaje basado en problemas se ha convertido en una de las metodologías docente más útiles a la hora de que los alumnos pongan en práctica los conocimientos y que desarrollen las destrezas y habilidades metodológicas, técnicas y profesionales. El grado en Publicidad y RRPP de la Universidad Camilo José Cela cuenta con una técnica didáctica, el Festival de Creatividad (Creadictos), que supone una forma de trabajo consistente en enfrentar a los alumnos a un problema o situación. Esto les va a permitir: comprender mejor ese problema/situación profesional, identificar principios que sustentan el conocimiento y alcanzar objetivos de aprendizaje especialmente relacionados con el razonamiento y el juicio crítico.

PALABRAS CLAVE: Metodologías Docentes - Aprendizaje basado en problemas Festival - Creatividad

\footnotetext{
${ }^{1}$ Autor correspondiente Laura Melendo Rodríguez-Carmona1. Universidad Camilo José Cela. Madrid. España. Correo: $\underline{\text { lmelendo@ucjc.edu }}$
} 


\title{
NEW LEARNING RESOURCES IN THE EUROPEAN FRAMEWORK: CREADICTOS (CREATIVITY FESTIVAT UCJC)
}

\begin{abstract}
One of the most useful teaching methodologies is problem based learning because it enables students to put theoretical knowledge into practice and to develop technical and professional skills. The Univertity Camilo José Cela offers this practical didactic technique through Creadictos as part of the degree in Public Relations and Advertising. Creadictos introduces an innovative working methodology which makes students face real problems and situations. As a result they are able to understand professional problems or situations from a practical perspective and, at the same time, identify principles that sustain knowledge attaining learning objectives related to reasoning and critical thinking.
\end{abstract}

KEY WORDS: Teaching Methodologies - Problem based learning - Festival Creativity

\section{INTRODUCCIÓN}

La Universidad Camilo José Cela tiene implantado el Grado en Publicidad y RRPP desde el curso 2010-2011. Dicho grado atiende a las directrices marcadas por el Espacio Europeo de Educación Superior (EEES). De esta manera, en la enseñanza presencial, las actividades formativas del estudiante se distribuyen entre el tiempo dedicado a las clases (teóricas y prácticas) y el trabajo autónomo.

Esta Universidad está sensibilizada en la implementación de nuevas metodologías docentes que posibiliten la adquisición de las competencias por parte de los alumnos. La clase magistral como metodología educativa queda reducida a un mínimo porcentaje y se complementa con otras metodologías: el aprendizaje basado en problemas, el contrato de aprendizaje, el método del caso y el portafolio del estudiante entre otras.

La realización de estas actividades puede combinar trabajo individual y de grupo garantizando, además, el desarrollo de las competencias genéricas recogidas en el perfil del título. Se trata de que el alumno ponga en práctica los conocimientos. Que desarrolle las destrezas y habilidades metodológicas, técnicas y profesionales. Así, los resultados de aprendizaje se refieren a saber aplicar los contenidos y dominar destrezas prácticas que contribuyen al desarrollo de las competencias profesionales. 


\section{METODOLOGÍA}

En este artículo desarrollamos un ejemplo de aprendizaje basado en problemas. En concreto se trata de la organización de un Festival de Creatividad (Creadictos) que involucra de manera transversal a alumnos de diferentes cursos.

Se trata de trabajar con un grupo grande autorizado que a su vez se subdivide en otros grupos posibilitado por el aprendizaje cooperativo, esto es, los alumnos de cursos superiores que ya han tenido la experiencia cursos anteriores sirven de mentores a los de cursos inferiores estimulando, así, la participación activa y la construcción de conocimiento por parte del grupo.

\section{Aprendizaje basado en problemas}

El Aprendizaje Basado en Problemas (ABP) como alternativa metodológica, estrategia o técnica didáctica, es una forma de trabajo consistente en enfrentar a los alumnos a un problema o situación que le va a permitir comprender mejor ese problema/situación profesional, identificar principios que sustentan el conocimiento y alcanzar objetivos de aprendizaje especialmente relacionados con el razonamiento y el juicio crítico.

El esquema básico de la metodología ABP consiste en el planteamiento de un problema o situación (normalmente definido por el docente y en ocasiones definido por los estudiantes) a través del cual se solicita de los estudiantes que, en grupos de trabajo, aborden de forma ordenada y desde un trabajo coordinado las diferentes fases que implica la resolución o desarrollo del trabajo en torno al problema o situación.

A modo de ejemplo, una secuencia de fases alrededor del trabajo en torno a determinado problema podría ser:

a) Delimitación precisa del problema o tarea a resolver.

b) Necesidad de búsqueda de información y recursos (búsqueda, acceso y validación de información).

c) Diseño de un plan de trabajo coordinado.

d) Desarrollo del plan.

e) Puesta en común de resultados o conclusiones.

f) Elaboración de un documento o informe común. 
Se trata de una metodología donde, dependiendo del nivel y preparación del estudiante, del carácter del problema o trabajo planteado, del tiempo y recursos disponibles, etc., la autonomía y tutorización de los grupos de trabajo será mayor o menor. Por otra parte dicha forma de trabajo no sólo trata de abordar objetivos relativos al conocimiento o dominio de una asignatura o determinada área de conocimientos, sino también el desarrollo de habilidades y competencias relativas a la capacidad de trabajar en equipo, de búsqueda y validación de información, de habilidades comunicativas, etc.

Es importante tener en cuenta dos aspectos básicos:

a) El docente en todo momento es un tutor/facilitador que ha de promover el trabajo colaborativo.

b) El objetivo no es resolver el problema sino que el problema es la causa para que los estudiantes consigan adquirir los objetivos de aprendizaje planteados en ese problema.

\section{ANÁLISIS Y DISCUSIÓN}

Creadictos es un festival de creatividad que se celebra en la Universidad Camilo José Cela de Madrid, en su campus de Villanueva de la Cañada.

Es promovido por la Facultad de Ciencias de la Comunicación en su interés de generar un evento que sirviese al mismo tiempo como elemento aglutinador de los diferentes títulos de la Facultad, actuando como enlace entre profesores y alumnos $\mathrm{y}$, al mismo tiempo, resolviendo una necesidad práctica convirtiendo a Creadictos en plataforma docente donde los alumnos pudiesen llevar a la práctica todo lo aprendido a lo largo de la carrera.

Su organización corre a cargo de Pícame, la Agencia Junior de Comunicación de la Facultad de Ciencias de la Comunicación, y del resto de alumnos de la titulación de Publicidad y RR.PP. Además, cuenta con la colaboración de los alumnos de Periodismo, Comunicación Audiovisual y Protocolo y del Laboratorio de Comunicación. Todo ello, como no, supervisado por los profesores de la Facultad.

En su organización también participan puntualmente otras facultades, departamentos y colaboradores externos de la universidad. Se celebra en el mes de mayo y en su agenda se incluyen conferencias, talleres, concursos, conciertos y actividades lúdicas. 


\subsection{Objetivos de creadictos}

El principal objetivo de Creadictos es normalizar las actividades prácticas que se realizan dentro de las asignaturas del plan de grado en Publicidad y Relaciones Públicas. Para ello, los profesores de diferentes asignaturas coordinan sus trabajos prácticos y los orientan a la organización del festival. Así los alumnos pueden poner en práctica lo aprendido en las aulas con un caso real.

Las asignaturas involucradas son las que se detallan a continuación, pertenecientes al segundo, tercer y cuarto curso:
a) Estrategias publicitarias
b) Creatividad publicitaria
c) Marketing
d) Técnicas de investigación de mercados
e) Teoría y Práctica de las relaciones públicas
f) Planificación de medios

Este festival es esperado por alumnos, profesores y antiguos alumnos durante todo el curso. Es una cita anual en la que todos se reencuentran fomentando el sentimiento de pertenencia a la UCJC.

También es un semillero de ideas. De este encuentro nacen muchas iniciativas que posteriormente se acaban instalando definitivamente en la agenda de la universidad. El concurso de pinchos de la Cátedra de Ferrá Adriá o la paella solidaria de la Oficina de Cooperación Universitaria, por ejemplo, fueron originalmente actividades dentro de la agenda del Festival y hoy han adquirido identidad propia y se desarrollan de manera independiente.

Cuenta con una campaña de comunicación diseñada por los propios alumnos y atiende al objetivo de difusión del evento en dos tipos de medios: medios externos y medios internos a la UCJC.

En cuanto a los medios externos, los alumnos contactan con los soportes especializados en publicidad y comunicación y medios locales, mediante nota de prensa. Suelen conseguir gran apoyo en ellos (Anuncios, El Publicista, marketing Directo, El programa de la Publicidad, Estrategias, Interactiva y Brandlife, entre otros, han publicado la nota de prensa). Los medios locales también se interesan por el festival (Villanueva al día, por ejemplo).

Esta repercusión en los medios permite a la UCJC ser protagonista en medios especializados del sector de la publicidad y la comunicación. De manera que, a través del Festival, la Universidad consigue notoriedad y contribuye a sus objetivos de promoción. 
Los medios internos de la Universidad, es decir, el periódico La Colmena, la emisora Onda Villanueva, y Media Campus TV de la UCJC, realizan una gran cobertura del festival. Durante el evento, profesores y alumnos de Comunicación Audiovisual y Periodismo retransmiten, comentan y hacen programas especiales, poniendo en práctica sus conocimientos adquiridos en el aula.

En la última edición, los alumnos del Grado en Protocolo y Organización de Eventos colaboraron de manera muy activa ayudando a sus compañeros en la organización de los acontecimientos más relevantes.

La financiación del festival también es responsabilidad de los alumnos que diseñan un dossier de patrocinio y contactan con diferentes entidades a fin de conseguir la captación de patrocinadores y colaboradores.

Deben estudiar los posibles patrocinadores, analizar su afinidad con el evento, contactar con ellos, y proponerles una contraprestación.

Esto ayuda a reducir el presupuesto en gran parte, y ofrece al alumno una experiencia muy gratificante al lograr cerrar acuerdos reales de colaboración.

\subsection{Competencias y resultados de aprendizaje}

Los resultados de aprendizaje a nivel genérico que se promueven con esta actividad son:

a) Pensamiento crítico

b) Creatividad e innovación

c) Toma de decisiones en situaciones nuevas

d) Desarrollar el aprendizaje autodirigido

e) Identificar, buscar y analizar información necesaria para temas particulares

f) Habilidades comunicativas

g) Habilidad para trabajar de manera colaborativa

h) Desarrollo de la capacidad de empatía

i) Confianza para hablar en público

j) Habilidad para identificar las propias fortalezas y debilidades. Tomar medidas necesarias para mejorar

k) Habilidad de organización y planificación

1) Habilidad para resolver problemas y tomar decisiones

m) Gestión eficiente del tiempo

n) Habilidad para la gestión económica y presupuestaria 


\subsection{Retrospectiva}

Si bien la temática general del festival siempre es la creatividad en la comunicación y la publicidad, cada edición se centra en un área en concreto. Son los alumnos los que escogen el eje de cada edición, su eslogan y objetivos.

A continuación se presentan las ediciones realizadas hasta la fecha:

CR3ADICTOS'04 "Agencias de Medios" se centró en la importancia de las agencias de medios dentro del proceso publicitario. Tuvo lugar en el año 2004 y su propósito fue afianzarse como festival de referencia en el mundo universitario.

En la segunda edición CR3ADICTOS”05 "Exprésate en futuro", se quiso acercar el mundo laboral a los estudiantes de publicidad.

CR3ADICTOS'06 tuvo como lema "La comunicación como arte, la creatividad como medio". Su objetivo era relacionar y hacer entender que los conceptos de creatividad y comunicación están íntimamente relacionados.

La cuarta, CR3ADICTOS'07, trató el eje "Deconstruye la publicidad". Este año insistió en la intención de mostrar todos los elementos que componen el proceso publicitario. El festival transcurrió al aire libre y destacó por la celebración del Foro Social Creadictos.

CR3ADICTOS'08 “Exprime tu mundo, Squeeze your world”, planteó una visión del mundo a través de la publicidad.

La siguiente edición, CR3ADICTOS’09, promovió una "Creatividad con conciencia". Se defendieron unos planteamientos creativos que apoyasen, fomentasen y respetasen la protección de los derechos humanos fundamentales y asegurasen que con sus fines no son cómplices en su vulneración.

En el año 2010 se planteó un nuevo reto: "AD2U”. Se hizo algo nunca antes visto en el mundo de la publicidad: los asistentes tuvieron que grabar tres spots en doce horas empleando la cámara de un teléfono móvil.

En la última edición, "CR3ADICTOS'11", el equipo de Pícame ha querido aprovechar el décimo aniversario de la UCJC para aglutinar a la comunidad universitaria, con el objetivo de fomentar el espíritu universitario. Se ha centrado en el lema "Planeta UCJC: cinco facultades, cinco continentes, diez años de la UCJC". 


\subsection{Público asistente}

El principal público objetivo del festival son los alumnos de la Facultad de Comunicación en particular y de la UCJC en general. Pero en cada edición se ha ido ampliando el target y abriéndose a otros grupos de interés.

Por lo tanto, en este encuentro se dan cita:

a) Alumnos de la Universidad Camilo José Cela

b) Jóvenes y futuros universitarios residentes en los municipios de la zona noroeste de Madrid interesados en el mundo de la Comunicación, la Publicidad y la Creatividad

c) Alumnos de otras universidades de Madrid, principalmente las más relacionadas con el mundo de la Comunicación (Complutense, Alfonso X, Antonio de Nebrija, UEM, Villanueva y Francisco de Vitoria, entre otras)

d) Profesores y PAS UCJC

e) Profesionales del sector

f) Amantes de la creatividad, el arte y la comunicación

\subsection{Plan de trabajo}

Los alumnos tienen un plan de trabajo definido desde inicio del curso. Este plan está organizado en varias fases y temporalizado:

\section{Fase inicial}

Primeras reuniones y creación del grupo de trabajo. La primera acción se centra en organizar el equipo internamente. Se realiza el reparto de responsabilidades y se elabora un organigrama del grupo responsable de la organización.

El grupo de trabajo se organiza por departamentos (producción, comunicación, patrocinios, diseño, conciertos y concursos, entre otros) y se nombran a dos alumnos coordinadores del evento.

\section{Fase estratégica}

Los alumnos deben escoger la temática, definir los objetivos de la edición y realizar la planificación en bruto de las actividades. Este pre-proyecto se recoge en un dossier que debe ser presentado a la junta de la facultad y aprobado ella.

También se empieza a trabajar en la imagen corporativa y comunicación del evento (incluye varios bocetos del cartel, del programa, de la web y del resto del material de comunicación). 
Los responsables de cada departamento presentan su planteamiento, ideas iniciales, objetivos y estrategia.

\section{Fase táctica}

La fase táctica se centra en los preparativos. En esta etapa se presenta el presupuesto final, se cierran los acuerdos con los proveedores, se confirman los conferenciantes y se pone en marcha la campaña de comunicación (producción y distribución), entre otros.

También se elabora un cronograma del evento para el equipo organizador. En él se contemplan todas las actividades, horarios y tareas y los responsables de su ejecución y control.

\section{Ejecución del Festival Creadictos}

En el mes de mayo tiene lugar el festival en el campus de Villanueva de la Cañada.Durante su celebración se emplean todas las instalaciones del campus: aulas, salón de grados, laboratorio, cafetería y zonas ajardinadas. La agenda se estructura en varias secciones: mañana, comida y tarde/noche. Por la mañana se realizan las actividades con carácter docente y educativo (conferencias, actividades y encuentros profesionales).

A medio día se realiza una actividad que fomente el compañerismo mediante la elaboración de una paella gigante o reparto de bocadillos en la pradera, por ejemplo. Para la tarde se reservan las actividades formativas de diálogo entre los alumnos y los profesores como los concursos y talleres. Al finalizar el día se celebra el fin de fiesta con música y conciertos a cargo de alumnos.

\section{Post-Evento}

Al finalizar el festival se envía una carta de agradecimiento a patrocinadores, se hacen las labores de post-producción del making-off y un balance de la repercusión en medios.

\section{Memoria}

Los alumnos deben realizar y entregar a sus profesores una memoria del evento. 


\section{CONCLUSIONES}

El ABP resulta útil en la medida que atiende a los siguientes objetivos:

a) Potenciar el desarrollo integral del estudiante (conocimientos, procedimientos, habilidades, actitudes y valores).

b) Fomentar una actitud positiva hacia su propio aprendizaje (respeto de la autonomía del estudiante).

c) Estimula el autoaprendizaje ya que el estudiante aprende a través de la propia experiencia adquirida durante la dinámica de trabajo.

d) Fomentar el trabajo en equipo.

e) Estimular la motivación.

f) Lograr un aprendizaje significativo.

g) Transferir el aprendizaje recibido a situaciones reales. Entre las ventajas de la aplicación del ABP podemos citar:

h) Potencia el compromiso, la responsabilidad y confianza en el trabajo en equipo.

i) Desarrollo de las habilidades cognitivas del estudiante (pensamiento crítico, análisis, síntesis).

j) Los estudiantes aprenden sobre su propio proceso de aprendizaje.

También encontramos una serie de inconvenientes o dificultades que hay que salvar en la implementación de la metodología ABP:

a) El estudiante implicado en este tipo de metodología ha de reunir una serie de condiciones: disposición a trabajar en equipo, creatividad, capacidad de tomar decisiones, habilidades comunicativas y de interacción personal, capacidad de analizar y sintetizar información, perspectiva crítica, etc...

b) El profesor también debe reunir una serie de características para usar esta metodología: conocimiento de la materia, conocer la metodología, conocer el rol que va a adoptar, conocimiento de técnicas de evaluación adecuadas a esta metodología, accesibilidad, liderazgo, capacidad de síntesis, potenciar la retroalimentación, desarrollar el pensamiento crítico, etc...

c) Requiere mucho tiempo de dedicación tanto para el docente (es costoso preparar los problemas) y coordinar el trabajo de los alumnos a la hora de lograr los aprendizajes.

d) El docente plantea una actividad y en torno a ella el estudiante construye su aprendizaje.

e) Resulta necesaria una participación activa del estudiante en el logro de los objetivos de aprendizaje (Aprendizaje centrado en el alumno).

f) Importancia del trabajo cooperativo. 
Por lo tanto, y como conclusión a todo lo hasta ahora expuesto, Creadictos puede ser considerado una plataforma docente y creativa. Se ha convertido en una técnica o metodología didáctica basada en problemas que promueve el aprendizaje del alumno que se enfrenta a problemas o situaciones reales. Esto le va a permitir comprender mejor ese problema/situación profesional, identificar principios que sustentan el conocimiento y alcanzar objetivos de aprendizaje especialmente relacionados con el razonamiento y el juicio crítico.

\section{REFERENCIAS}

Arceo Vacas, J. L. (2003). Organización, Medios y Técnicas en Relaciones Públicas. Madrid: ICIE-UCM.

Barquero, J. D. (1999). Manual de Relaciones Públicas empresariales e institucionales. Barcelona: Gestión 2000.

Benito, Á. \& Cruz, A. (2007). Nuevas claves para la docencia universitaria en el espacio europeo de educación superior. Madrid: UEM.

Escribano, A. \& Del Valle, A. (2008). El Aprendizaje Basado en Problemas. Una propuesta metodológica en Educación Superior. Madrid: Narcea

Exley, K. \& Dennick, R. (2007). Enseñanza en pequeños grupos en Educación Superior. Tutorías, seminarios y otros agrupamientos. Madrid: Narcea

Fuente Lafuente, C. (2002). Técnicas de organización de actos. Madrid: Ediciones Protocolo.

Fuente Lafuente, C. (2005). Manual práctico para la organización de eventos. Madrid: Ediciones Protocolo.

Fuente Lafuente, C. (2007). Protocolo para eventos. Madrid: Ediciones Protocolo.

Lopez Noguero, F. (2007). Metodología participativa en la Enseñanza Universitaria. Madrid: Narcea

Miguel Díaz, M. (2006). Metodologías de enseñanzas y aprendizaje para el desarrollo de competencias: Orientaciones para el profesorado universitario ante el Espacio Europeo de Educación Superior. Madrid: Alianza Editorial S.A.

Sánchez Guzmán, J. R. (2005). Lo fundamental del marketing. Madrid: Grupo Konecta.

Seitel, F. P. (2002). Teoría y práctica de las Relaciones Públicas. Madrid: Pearson Prentice Hall. 


\section{Laura Melendo Rodríguez-Carmona}

Doctora en Ciencias de la Información, es profesora de la Facultad de Ciencias de la Comunicación en la Universidad Camilo José Cela. Su área de conocimiento se centra en el proceso de planificación y medios de comunicación publicitarios. Además de su labor docente, realiza la coordinación de las nuevas tecnologías aplicadas a la docencia en dicha facultad. Realiza colaboraciones con EAE Madrid y con el Colegio Oficial de Sociólogos y Politólogos de Madrid.

\section{Eva García-Montero.}

Doctora en Ciencias Sociales por la Universidad Camilo José Cela, licenciada en Psicología Industrial por la UNED y licenciada en Publicidad y Relaciones Públicas por la Universidad Complutense. También cuenta con un título de Especialista en Gestión Publicitaria por esta última Universidad. Ha desarrollado su carrera profesional en agencias de publicidad (McCann-Erickson y J. Walter Thompson) y departamentos de marketing de empresas, lo que compagina con su labor como docente en la Universidad Camilo José Cela de Madrid. Es profesora invitada en Plantjin I Hogeschool Amberes (Bélgica) y Artevelde Hogeschool Gante (Bélgica). 